\title{
Stimulation of the autonomic nervous system in colorectal surgery: a study protocol for a randomized controlled trial
}

\author{
Tim MP Berghmans ${ }^{1}$, Karel WE Hulsewé ${ }^{2}$, Wim A Buurman ${ }^{3}$ and Misha DP Luyer ${ }^{4^{*}}$
}

\begin{abstract}
Background: Postoperative ileus (POI) is a well-known complication of abdominal surgery and is considered to be caused by a local inflammation in the gut. Previously it has been shown that both local and systemic inflammation can be reduced by stimulation of the autonomic nervous system via lipid rich nutrition. Stimulation of the autonomic nervous system releases acetylcholine from efferent vagal nerve endings that binds to nicotinic receptors located on the inflammatory cells leading to a decrease of pro-inflammatory mediators. Besides administration of nutrition there are other ways of stimulating the autonomic nervous system such as gum chewing.

Methods/design: This prospective, placebo-controlled randomized trial will include 120 patients undergoing colorectal surgery which are randomized for gum chewing preoperatively and in the direct postoperative phase or a placebo. Postoperative ileus will be assessed both clinically by time to first flatus and time to first defecation and by determination of gastric motility using ultrasound to measure dimensions of the antrum. Furthermore the inflammatory response is quantified by analyzing pro-inflammatory mediators. Finally, markers of gut barrier integrity will be measured as well as occurrence of postoperative complications.

Discussion: We hypothesize that chewing gum preoperatively and in the direct postoperative phase in patients undergoing colorectal surgery dampens local and systematic inflammation, via activation of the autonomic nervous system. Down-regulation of the inflammatory cascade via stimulation of the vagus nerve will ameleriote POI and enhance postoperative recovery.
\end{abstract}

Trial registration: NTR2867

Keywords: Postoperative ileus, Chewing gum, Inflammation, Colorectal surgery, Autonomic nervous system

\section{Background}

All intra-abdominal surgical procedures, even minimally invasive, are followed by a transient episode of gastrointestinal hypomotility making postoperative ileus (POI) a common clinical phenomenon after abdominal surgery. Therefore it has been suggested to accept POI as an obligatory physiological response of the intestine when performing abdominal surgery [1]. However, POI clearly has a significant impact on patient morbidity and results in a prolonged hospital stay and significantly contributes to

\footnotetext{
* Correspondence: misha.luyer@catharina-ziekenhuis.nl

${ }^{4}$ Department of Surgery, Catherina Hospital Eindhoven, PO Box 13505602 ZA, Eindhoven, The Netherlands

Full list of author information is available at the end of the article
}

annual healthcare costs [1]. Implementation of fast-track colorectal surgical programs have already shown promising results in reducing overall hospital stay by epidural analgesia, earlier nutrition, and mobilization after surgery [2]. Further reduction of this postoperative transient episode of gastrointestinal hypomotility will add to the enhanced recovery after colorectal surgery.

Research has shown that the extent of POI is influenced by the degree of surgical trauma, bowel manipulation and duration of surgery [3]. The inflammatory response is regarded to play a key role in the etiology of POI next to factors as sympathetic neural reflexes and release of hormones and neurotransmitters. It is thought that handling of the intestine during abdominal surgery

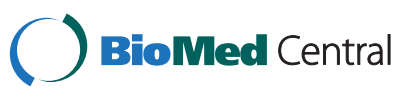


triggers the inflammatory cascade leading to local inflammation in the intestinal muscular layer and influx of leucocytes [4]. This local inflammation is important in the development of gastrointestinal hypomotility after abdominal surgery and thus POI $[1,4,5]$.

Previous studies have shown that vagus nerve stimulation ameliorates the local and systemic inflammatory response by binding of acetylcholine to $\alpha 7$ nicotinic ACh receptors (nAChrR) expressed on macrophages resulting in downregulation of tissue macrophage reactivity and cytokine release [6,7]. This neural feedback loop can be triggered mechanically, pharmacologically, or in a physiological way via administration of enteral nutrition leading to an instant reduction of the inflammatory cascade. In experimental rat models we have previously demonstrated that preoperative administration of lipid-enriched enteral nutrition strongly attenuates the inflammatory response and reduces POI via release of cholecystokinin and activation of afferent vagal fibers $[8,9]$. However, clinical research is necessary to substantiate these effects in humans.

Activation of the autonomic nervous system can also be established by sham-feeding such as chewing gum [10-12]. Several studies have shown favorable effects of chewing gum after surgery for POI, measured as time to flatus and overall hospital stay [13-15]. However, the underlying mechanism remains unclear. Furthermore, based on experimental data previously referred to, chewing gum before the surgery would be expected to be most effective whereas. However, until now all studies administered chewing gum in the postoperative phase only.

In this study we investigate the effect of perioperative gum chewing on postoperative inflammatory mediators and evaluate clinical parameters such as POI in patients undergoing colorectal surgery.

\section{Study objectives}

The primary objective of this study is to determine the effects of gum chewing in the perioperative phase on the extent of POI, overall hospital stay, and morbidity and mortality. The secondary objective is to determine the effects of gum chewing in the perioperative phase on postoperative inflammation and tissue damage.

\section{Methods and design}

This trial is designed as a prospective placebo-controlled randomized trial in which the effect of chewing gum before and directly after colorectal surgery is compared to a placebo.

\section{Study population}

Patients older than 18 years of age undergoing elective colorectal surgery are eligible for inclusion. In total 120 patients will be included. Patients are excluded in case of peritoneal carcinomatosis, inflammatory bowel disease, previous gastric or esophageal surgery (due to vagus nerve injury), a pre-existent ileostoma, allergy for mint, or gut motility influencing agents (for example, tricyclic antidepressants). Furthermore, patients with disturbance of the acetylcholine metabolism in any way, such as neurological diseases, medication (SSRI), or depression will be excluded.

\section{Participating centers}

Patients eligible for enrolment will be included in two hospitals in the Netherlands; Orbis Medical Center in Sittard and Catharina Hospital Eindhoven.

\section{Randomization}

After obtaining written informed consent patients will be randomly assigned to one of two groups by randomization software (TenAlea software adapted for minimization randomization). Based on this output a sequentially numbered, opaque, sealed envelope will be opened by which the allocation is revealed. Patients are either allocated to the experimental group and receive chewing-gum preoperatively and directly postoperative until start of enteral nutrition. The control group receives a placebo (dermal patch) for the same period, which does not contain any active ingredient, although patients are told otherwise. The dermal patch was chosen as placebo since mimicking the chewing of chewing gum in any other way would function as sham-feeding and thereby contaminate the results. The dermal patch can be considered comfortable, easy to use, and non-invasive, and serves the placebo purpose of patients assuming the patch to contain an active ingredient. Opening of the sealed envelopes and the administration of the intervention or the placebo is performed by nursing staff not participating in this trial. Ethical approval for this study was granted by the Medical Ethics Committee Atrium-Orbis-Zuyd (Heerlen, the Netherlands).

\section{Study outline}

All subjects are allowed to drink clear fluids up to $2 \mathrm{~h}$ before surgery. Patients allocated to the experimental group start chewing gum $3 \mathrm{~h}$ preoperatively until surgery, $3 \mathrm{~h}$ postoperatively gum chewing is continued. Though patients are free to chew the gum to their own liking during these periods, they are being encouraged to chew the gum as often as possible. Gum chewing is discontinued at the moment at which normal solid food intake is being tolerated. The control group will receive a dermal patch at the same moments as the experimental group and are instructed not to chew any gum pre- or postoperatively. The patch will be placed at a standardized location in the lumbar region. Both groups receive the same fast-track surgical protocol as any other patients undergoing colorectal surgery in our center. At moment of inclusion, consent is obtained and patient information will be given in which both the gum chewing as the placebo (dermal patch) are described as being possibly 
effective. The expected effective intervention (gum chewing) and definitive conclusion will not be revealed until completion of the trial.

Blood and urine samples will be collected at several predefined moments in relation to the incision time of the procedure. Tissue samples from the resected colorectal segment are collected during the surgical procedure for immunohistochemistry, Western blot, and polymerase chain reaction (PCR) analysis. When collected all samples are stored at $-80{ }^{\circ} \mathrm{C}$ until further analysis. The inflammatory response is measured by analyzing the blood plasma and tissue samples for inflammatory mediators (amongst which TNF-alpha and interleukins such as IL-6 and IL-8). Intestinal tissue damage is measured by analysis of several markers for intestinal damage in blood and tissue samples such as intestinal fatty acid binding protein (I-FABP).

Patient characteristics and clinical parameters, such as vomiting and time to return of a normal gastrointestinal transit, are registered in an electronic database.

On admission, a quality of life checklist (EORTC QLQ C-30) for cancer patients is filled out, which is repeated at time of discharge. Patients are ready for discharge according to known criteria as in fast-track surgery [16,17]. At the second postoperative day gastrointestinal transit is assessed by ultrasonography by a standard protocol.

\section{Antrum measurements}

POI is often measured clinically based on parameters such a time to first flatus and first defecation. These are more or less subjective parameters of gastric motility [14]. To provide an objective and precise parameter for gastric motility an ultrasound of the gastric antrum is performed in the evening of the second postoperative day. Previous studies have already shown antrum measurements to be representative for determining gastric emptying and thereby gastric motility [18-20]. In short, subjects receive a standardized meal after which antrum measurements are done. All results of these measurements are registered in an electronic database.

\section{Statistical analysis}

Sample size is calculated with a Power analysis and was aimed at POI and length of stay. We considered a decrease of 1 day of admission from the average 5 days for current fast-track protocols in our center to be clinically relevant. To achieve a power of 0.9 with an alpha of 0.05 and a beta of 0.1 for a decrease of 1 day of the mean length of stay each group needs to consist of 60 subjects when performing a unpaired $T$-test. The analysis will be done according to the intention-to-treat principle. When using POI as primary outcome parameter for the Power analysis slightly fewer patients would be required per group due to the larger effect size. With the current sample size, both parameters are adequately powered.

\section{Discussion}

POI is defined as delayed return of gastrointestinal motility occurring after abdominal surgical procedures. Recent advancements in perioperative care significantly enhanced patient recovery and reduced the overall hospital stay. However POI remains an important clinical problem without a therapeutic solution and interventions rely primarily on supportive measures [9,21].

Furthermore, POI also contributes to a prolonged length of stay that leads to increased healthcare costs [22]. The annual costs related to POI have been estimated to be as much as $\$ 1.47$ billion annually in the US [1,22]. Reducing POI and thereby diminishing length of stay will result in a significant decrease of healthcare costs.

It is thought that the inflammatory response within the muscular layer of the gut which is triggered by intestinal manipulation plays a key role in the etiology of POI. Reduction of this postoperative transient episode of hypomotility of the gastrointestinal tract by dampening the inflammatory response seems a promising approach in ameliorating POI. We have already demonstrated that administration of enteral nutrition dampens the inflammatory response in experimental models [8]. This positive feedback mechanism involves release of cholecystokinin leading to activation of the autonomic nervous system. Sham-feeding is a potential other way of stimulating the autonomic nervous system after abdominal surgery.

Sham-feeding by chewing gum has been shown to ameliorate POI following gastrointestinal surgery when started after surgery [13-15]. However, the mechanism underlying the beneficial effect of gum chewing on POI remains elusive. Based on experimental studies, it may well be that activation of the autonomic nervous system leading to an inhibition of the inflammatory response plays an important role. In this light, chewing gum before the surgery would be expected to be most effective.

Therefore, our hypothesis is that chewing gum before and directly after colorectal surgery will dampen the inflammatory response, both locally and systematically, via stimulation of afferent vagal nerve fibers. Hereby, POI will be ameliorated and patients' recovery will be enhanced.

This study was designed to investigate the effect of chewing gum on POI, the inflammatory response, and length of stay.

\section{Trial status}

Ongoing trial, patient recruitment.

\section{Abbreviations}

POI: Postoperative ileus.

\section{Competing interests}

The authors declare that they have no competing interests.

Acknowledgements

This trial is self-supporting; no funding is received. 


\section{Author details}

${ }^{1}$ Faculty of Medicine, Maastricht University, Maastricht ER 6229, The Netherlands. ${ }^{2}$ Department of Surgery, Orbis Medical Centre, Sittard MB 6130 The Netherlands. ${ }^{3}$ Department of Surgery, Maastricht University, Maastricht, ER 6229, The Netherlands. ${ }^{4}$ Department of Surgery, Catherina Hospital Eindhoven, PO Box 13505602 ZA, Eindhoven, The Netherlands.

\section{Authors' contribution}

TB drafted the manuscript. ML authored the writing of the manuscript. All authors participated in the design of the study and are local investigators at the participating centers. All authors read, edited, and approved the final manuscript.

Received: 21 December 2011 Accepted: 27 June 2012

Published: 27 June 2012

\section{References}

1. Boeckxstaens GE, de Jonge WJ: Neuroimmune mechanisms in postoperative ileus. Gut 2009, 58:1300-1311.

2. Gouvas N, Tan E, Windsor A, Xynos E, Tekkis PP: Fast-track vs standard care in colorectal surgery: a meta-analysis update. Int J Colorectal Dis 2009, 24:1119-1131.

3. Turler A, Moore BA, Pezzone MA, Overhaus M, Kalff JC, Bauer AJ: Colonic postoperative inflammatory ileus in the rat. Ann Surg 2002, 236:56-66.

4. Kalff JC, Carlos TM, Schraut WH, Billiar TR, Simmons RL, Bauer AJ: Surgically induced leukocytic infiltrates within the rat intestinal muscularis mediate postoperative ileus. Gastroenterology 1999, 117:378-387.

5. Luckey A, Livingston E, Tache Y: Mechanisms and treatment of postoperative ileus. Arch Surg 2003, 138:206-214.

6. Tracey KJ: Physiology and immunology of the cholinergic antiinflammatory pathway. J Clin Invest 2007, 117:289-296

7. Van Der Zanden EP, Boeckxstaens GE, de Jonge WJ: The vagus nerve as a modulator of intestinal inflammation. Neurogastroenterol Motil 2009, 21:6-17.

8. Luyer MD, Greve JW, Hadfoune M, Jacobs JA, Dejong CH, Buurman WA: Nutritional stimulation of cholecystokinin receptors inhibits inflammation via the vagus nerve. J Exp Med 2005, 202:1023-1029.

9. Lubbers T, Luyer MD, de Haan JJ, Hadfoune M, Buurman WA, Greve JW: Lipid-rich enteral nutrition reduces postoperative ileus in rats via activation of cholecystokinin-receptors. Ann Surg 2009, 249:481-487.

10. Wojdemann M, Traberg P, Stadil F, Sternby B, Larsen S, Hilsted L, Olsen O: Effect of sham feeding and acute suppression of acid secretion on human gastric lipase secretion. Am J Gastroenterol 1998, 93:244-248.

11. Helman CA: Chewing gum is as effective as food in stimulating cephalic phase gastric secretion. Am J Gastroenterol 1988, 83:640-642.

12. Lunding JA, Nordstrom LM, Haukelid AO, Gilja OH, Berstad A, Hausken T: Vagal activation by sham feeding improves gastric motility in functional dyspepsia. Neurogastroenterol Motil 2008, 20:618-624.

13. de Castro SM, van den Esschert JW, van Heek NT, Dalhuisen S, Koelemay MJ, Busch OR, Gouma DJ: A systematic review of the efficacy of gum chewing for the amelioration of postoperative ileus. Dig Surg 2008, 25:39-45.

14. Noble EJ, Harris R, Hosie KB, Thomas S, Lewis SJ: Gum chewing reduces postoperative ileus? A systematic review and meta-analysis. Int J Surg 2009, 7:100-105.

15. Fitzgerald JE, Ahmed I: Systematic review and meta-analysis of chewinggum therapy in the reduction of postoperative paralytic ileus following gastrointestinal surgery. World J Surg 2009, 33:2557-2566.

16. Maessen J, Dejong $\mathrm{CH}$, Hausel J, Nygren J, Lassen $\mathrm{K}$, Andersen J, Kessels AG, Revhaug A, Kehlet $H$, Ljungqvist O, Fearon KC, von Meyenfeldt MF: A protocol is not enough to implement an enhanced recovery programme for colorectal resection. Br J Surg 2007, 94:224-231.

17. Maessen JM, Dejong CH, Kessels AG, von Meyenfeldt MF: Length of stay: an inappropriate readout of the success of enhanced recovery programs. World J Surg 2008, 32:971-975.

18. Ricci R, Bontempo I, Corazziari E, La Bella A, Torsoli A: Real time ultrasonography of the gastric antrum. Gut 1993, 34:173-176.

19. Darwiche G, Almer LO, Bjorgell O, Cederholm C, Nilsson P: Measurement of gastric emptying by standardized real-time ultrasonography in healthy subjects and diabetic patients. J Ultrasound Med 1999, 18:673-682

20. Haruma K, Kusunoki H, Manabe N, Kamada T, Sato M, Ishii M, Shiotani A, Hata J: Real-time assessment of gastroduodenal motility by ultrasonography. Digestion 2008, Suppl 1:48-51.
21. Mattei $P$, Rombeau JL: Review of the pathophysiology and management of postoperative ileus. World J Surg 2006, 30:1382-1391.

22. Asgeirsson T, El-Badawi KI, Mahmood A, Barletta J, Luchtefeld M, Senagore AJ: Postoperative ileus: it costs more than you expect. J Am Coll Surg 2010, 210:228-231.

doi:10.1186/1745-6215-13-93

Cite this article as: Berghmans et al: Stimulation of the autonomic nervous system in colorectal surgery: a study protocol for a randomized controlled trial. Trials 2012 13:93.

\section{Submit your next manuscript to BioMed Central and take full advantage of:}

- Convenient online submission

- Thorough peer review

- No space constraints or color figure charges

- Immediate publication on acceptance

- Inclusion in PubMed, CAS, Scopus and Google Scholar

- Research which is freely available for redistribution

Submit your manuscript at www.biomedcentral.com/submit
C BioMed Central 